\title{
Palliative care in amyotrophic lateral sclerosis: a review of current international guidelines and initiatives
}

\author{
Peter Bede, ${ }^{1}$ David Oliver, ${ }^{2,3}$ James Stodart, ${ }^{4}$ Leonard van den Berg, ${ }_{1}^{5}$ \\ Zachary Simmons, ${ }^{6}$ Doiminic Ó Brannagáin, ${ }^{7,8}$ Gian Domenico Borasio, ${ }^{9,10}$ \\ Orla Hardiman ${ }^{1,11}$
}

\author{
See Editorial Commentary, \\ p 356 \\ ${ }^{1}$ Trinity College, Dublin, Ireland \\ ${ }^{2}$ Wisdom Hospice, Rochester, \\ UK \\ ${ }^{3}$ Centre for Professional \\ Practice, University of Kent, \\ Canterbury, UK \\ ${ }^{4}$ Wesley Hospital, Brisbane, \\ Queensland, Australia \\ ${ }^{5}$ Department of Neurology, \\ University Medical Centre \\ Utrecht, Heidelberglaan, \\ Utrecht, The Netherlands \\ ${ }^{6}$ Penn State Hershey Medical \\ Center, Department of \\ Neurology, Hershey, \\ Philadelphia, USA \\ ${ }^{7}$ Royal College of Surgeons in \\ Ireland, Dublin, UK \\ ${ }^{8}$ Dochas Centre, Our Lady of \\ Lourdes Hospital, Drogheda, Co \\ Louth, Ireland \\ ${ }^{9}$ Interdisciplinary Centre for \\ Palliative Medicine, Munich \\ University Hospital, Munich, \\ Germany \\ ${ }^{10}$ Department of Neurology, \\ University of Munich, Munich, \\ Germany \\ ${ }^{11}$ National Neuroscience Centre, \\ Beaumont Hospital, Dublin, \\ Ireland
}

Correspondence to Dr P Bede, Smurfit Building, Clinical Research Centre, Beaumont Hospital, Dublin 9 Dublin, Ireland: bedepeter@hotmail.com

Received 16 October 2010 Revised 8 December 2010 Accepted 17 December 2010 Published Online First 5 February 2011

\begin{abstract}
Background Amyotrophic lateral sclerosis (ALS) is a relentlessly progressive neurodegenerative condition. Optimal management requires a palliative approach from diagnosis with emphasis on patient autonomy, dignity and quality of life.
\end{abstract}

Objective To conduct a systematic analysis of the type level and timing of specialist palliative care intervention in ALS.

Results Despite an international consensus that ALS management should adopt a multidisciplinary approach, integration of palliative care into ALS management varies considerably across health care systems. Late referral to palliative services in ALS is not uncommon and may impact negatively on the quality of life of ALS patients and their caregivers. However, common themes and principles of engagement can be identified across different jurisdictions, and measurement systems have been established that can assess the impact of palliative care intervention.

Conclusions There is considerable evidence that palliative care intervention improves quality of life in patients and carers. International consensus guidelines would assist in the development of a framework for active palliative care engagement in ALS and other neurodegenerative diseases.

\section{INTRODUCTION}

Expansion of palliative care into non-malignant conditions has contributed to improvements in quality of life, symptom control and caregiver burden in neurodegenerative conditions such as amyotrophic lateral sclerosis (ALS). ${ }^{1}$ The point at which referral to palliative services occurs varies considerably across different countries. Early involvement and a dynamic model of intervention have been repeatedly suggested for ALS but few countries have developed integrated pathways of care. The objective of this review is to identify and examine the existing guidelines for palliative care in ALS, focusing on time of referral, triggers of referral and coordination of care.

\section{METHODS}

Publications were searched during a 4 month period between April 2010 and July 2010. Only articles published in English were reviewed. Further articles were identified through reviews of the references of these articles. Reports from meetings were also used if they presented relevant information.
The literature search was performed using PubMed and Medline with the following keywords: motor neuron disease (MND), amyotrophic lateral sclerosis (ALS), neurodegenerative disorder, symptomatic management, palliative care, guideline, pathway, framework, policy and algorithm.

\section{RESULTS}

\section{Palliative care in ALS}

It is generally recognised that optimal ALS management should adopt a multidisciplinary approach that includes palliative care. ${ }^{2}$ There is considerable evidence that multidisciplinary care improves both survival and quality of life in ALS. ${ }^{3} 4$ It is also recognised that effective communication between the multidisciplinary ALS team, the primary care physician and the palliative care team is essential, as patients with end stage ALS frequently develop distressing symptoms over a short period of time.

\section{Timing of specialist palliative care intervention in ALS}

The European Federation of Neurological Sciences taskforce $^{6}$ recommends that a palliative care approach should be adopted from the time of diagnosis and that palliative care should be available within the local community. Early referral to palliative services in advance of the terminal phase of ALS is recommended, as sufficient time is required to establish relationships with the staff and to address end of life issues. This is of particular importance as speech and communication are often limited in the later stages of disease.

In practice however, these recommendations are rarely observed and there is currently no widely accepted international standard for the initiation of palliative care intervention. Management of the terminal phase of ALS has been reported to be unsatisfactory in $33 \%$ of cases in northern Europe and in $61 \%$ of cases in southern Europe. ${ }^{7}$ In the UK, only $30 \%$ of palliative units provide ALS care, and only $8 \%$ are involved from the time of diagnosis. ${ }^{8}$

In the USA, stringent criteria are applied by Medicare for access to hospice based palliative care. The criteria include critically impaired breathing, rapid progression and severe nutritional impairment or rapid progression with life threatening complications. These guidelines have been widely criticised $^{9}$ as many patients who do not meet the Medicare hospice criteria die in less than 6 months. 
In one study, ${ }^{10}$ only five of 97 ALS patients who were accepted into hospice care from a multidisciplinary ALS centre met the Medicare criteria.

The US based ALS Peer Workgroup ${ }^{11}$ has suggested a number of specific triggers for hospice referral of ALS patients (box 1).

Referral triggers tend to be based on the more typical symptoms of ALS, such as respiratory and nutritional compromise. Rare, but distressing, complications of ALS such as severe pain of uncertain aetiology, frozen shoulder and painful mouth ulcers are frequently omitted from these guidelines, despite the fact that palliative teams have considerable experience in alleviating these symptoms.

Most ALS patients die at home. Where available, community palliative care programmes can provide valuable home based services and short term respite in the later stages of the disease. These services are not ubiquitous, and many ALS patients have no interaction with palliative services during their illness. ${ }^{12}$ In a survey of 215 Irish patients with ALS, only $28 \%$ successfully accessed palliative services. ${ }^{13}$ Perceived barriers to specialist palliative management of patients with non-malignant and neurodegenerative conditions include limited resources and lack of familiarity of providers with the disease trajectory of ALS ${ }^{14}$ (box 2).

Other important differences across healthcare systems have been noted in the management of $\mathrm{ALS}^{15}$ (table 1). These are primarily a function of complex local financial, legal and cultural factors.

There is evolving evidence that access to additional assistive equipment might sometimes be limited once the patient is formally receiving palliative care. Ideally, palliative care should be integrated into the larger multidisciplinary care, and initiation of hospice care should not limit availability of supportive treatment such as gastrostomy and non-invasive ventilation.

\section{End of life and advance directives}

Advance directives provide patients with the option to exercise autonomy regarding preferred end of life management strategies. The legal validity of advance directives varies from country to country.

Advance directives require a level of intellectual competence that enables processing of relevant information and appreciation of the consequences of decision making. Advance planning is

\section{Box 1 Suggested triggers for hospice referral by the} Amyotrophic Lateral Sclerosis Peer Workgroup

1. Forced vital capacity (FVC): $60 \%$ predicted (or rapid decline in FVC (more than $20 \%$ ) over $2-3$ months) or

2. Clinical signs or clinical symptoms of respiratory insufficiency or

3. Respiratory weakness requiring non-invasive positive pressure ventilation

or

4. Nutritional decline requiring enteral feeding or

5. Severe pain or psychosocial distress requiring intensive palliative care interventions (including opioid medication) or

6. Rapidly progressive paralysis (over $2-3$ months) in two body regions

\section{Box 2 Key barriers to palliative care intervention in} non-malignant conditions in Ireland

1. Unpredictable non-cancer disease trajectory

2. Lack of defined referral criteria for non-malignant conditions

3. Lack of non-cancer disease specific expertise

4. Limited resources and high number of potential non-cancer referrals

considered in three main formats: (a) an advance statement, made by the person when still competent; (b) an advance statement refusing treatment-this is legally binding in many jurisdictions; and (c) a lasting power of attorney, which defines a proxy to make decisions.

Advance directives are believed to be useful in 78\% of European centres although only $30 \%$ of patients actually complete them.

\section{Caregiver support}

Effective palliative frameworks need to address caregiver needs. There is evolving evidence that structured caregiver support, including counselling, support groups and a crisis management system, should be available for caregivers. ${ }^{16}$ Key barriers to comprehensive carer support include the time constraints of healthcare professionals and caregivers, lack of administrative support, lack of trained personnel and financial barriers. ${ }^{17}$

Few studies have approached the optimal timing of hospice referral from the carers' point of view. A prospective interview based study ${ }^{18}$ showed that the shorter the length of the stay in a hospice, the more likely the families felt that enrolment to the hospice had occurred too late.

\section{Impact of cognitive and behavioural impairment on ALS management}

Up to $40 \%$ of patients with ALS have evidence of cognitive impairment. ${ }^{1920}$ Despite the published consensus criteria ${ }^{21}$ for the diagnosis of cognitive and behavioural syndromes in ALS, the practical application of the criteria can be problematic. Formal neuropsychological testing may be particularly difficult due to disease specific factors such as dysarthria, physical disability, hypercapnia and medication effects. Currently, different batteries of neuropsychological tests are used in different centres. Most experts agree that a specialist clinical neuropsychologist should be available as part of the multidisciplinary ALS team. ${ }^{22}$ Short cognitive screening tests have been generated recently but have not yet been validated. ${ }^{23}$

Recognition of cognitive impairment is important because survival is significantly shorter among ALS patients with

Table 1 Cross cultural differences in amyotrophic lateral sclerosis management (Oliver et $\mathrm{al}^{15}$ )

\begin{tabular}{llll}
\hline Medical and palliative intervention & UK & USA & Japan \\
\hline Gastrostomy & $23 \%$ & $50 \%$ & $35 \%$ \\
$\quad$ Mean duration of gastrostomy feeding (months) & 11 & 18 & 17 \\
Non-invasive ventilation & $13 \%$ & $20 \%$ & $36 \%$ \\
$\quad$ Mean duration of non-invasive ventilation (months) & 10 & 5 & 5 \\
Invasive ventilation & $4 \%$ & 0 & $9 \%$ \\
$\quad$ Mean duration of invasive ventilation (months) & 10 & 0 & 80 \\
End of life and advance directive discussions & $23 \%$ & $80 \%$ & Not recorded \\
$\quad$ Timing of discussion (mean number of months & 5 & 10 & \\
prior to death) & & & \\
\hline
\end{tabular}


frontotemporal dementia ${ }^{24}$ and ALS patients with executive dysfunction (Elamin M, submitted 2011). Those with mild cognitive changes are also less likely to choose long term mechanical ventilation. ${ }^{25}$ Compliance with non-invasive ventilation is adversely affected by cognitive impairment. ${ }^{24}$ Chio and colleagues ${ }^{26}$ have recently demonstrated that behavioural deficits in ALS have a significant negative impact on caregivers' quality of life and increase carer burden considerably. ALS patients with executive dysfunction are also at higher risk of falls, choking episodes and injuries. They show poorer compliance with walking aids, percutaneous endoscopic gastrostomy or radiologically inserted gastrostomy feeding tubes and other safety devices.

It is likely that the concepts learnt from the palliative management of other neurodegenerative conditions such as advanced dementias can be applied in ALS. Insistence on noninvasive ventilation and feeding tube placement in the presence of significant behavioural and cognitive deficits can be inappropriate and should be carefully considered on an individual basis. Recent data indicate that cognitive change must be considered when approaching difficult decisions about management in the later stages of the disease. ${ }^{27}$ However, the full implications of ALS related cognitive decline on disease management remain to be formally established.

\section{Timing of 'end of life discussions'}

The optimal timing of end of life discussions in ALS is relatively well defined in the international literature. 'Trigger points' for discussion of end of life issues in ALS have been suggested by a number of authors. ${ }^{1128}$ These include the presence of patient distress, the evolution of disease or the expressed desire of the patient to discuss these issues (box 3 ).

\section{Coordination of ALS care in different healthcare systems}

International evidence based palliative models for ALS can be developed by integration of successful regional initiatives and identification of areas requiring further research. Although access to palliative services, referral habits and coordination of care vary considerably from country to country, common themes and principles can be identified.

\section{The Netherlands}

The Dutch protocol for ALS management has been recently published $^{29}$ (box 4). Coordination of care is clearly defined in the different phases of ALS. The protocol divides the disease into

\section{Box 3 Triggers of end of life discussions in amyotrophic} lateral sclerosis

1. The patient opens the discussion-for end of life information and/or interventions (elicited or spontaneous, verbal or non-verbal).

2. The presence of severe psychological, social or spiritual distress or suffering

3. The presence of pain requiring high dosages of analgesic medications

4. The presence of dysphagia requiring feeding tube

5. The presence of dyspnoea, symptoms of hypoventilation or a forced vital capacity of $50 \%$ or less

6. Loss of function in two body regions (regions include bulbar, arms and legs)
Box 4 The governing principles of the Dutch protocol for amyotrophic lateral sclerosis management

1. Autonomy of the amyotrophic lateral sclerosis patient

2. Medical information should be given in time for adequate decision making

3. The consultant in rehabilitation medicine should guarantee optimal symptomatic rehabilitation care through to palliative care in the terminal phase

4. Advance health directives should be repeatedly discussed

diagnostic, rehabilitation and terminal phases and designates a care coordinator for each. In the diagnostic phase, the neurologist is the care coordinator; in the rehabilitation phase, the consultant in rehabilitation medicine is the care coordinator; while the general practitioner is the main care coordinator in the terminal phase.

In a prospective Dutch study, ${ }^{3}$ patients treated by multidisciplinary ALS care teams had a significantly better psychological quality of life than those looked after by a general care group.

\section{UK}

A Neurological Conditions Policy Group was set up in 2004 by the National Council for Palliative Care to evaluate good practice examples and to identify effective service models. Gaps in coordination between different services and an overlap in their activities have been identified and a patient pathway has been established. ${ }^{30}$ This describes specific indicators for referral and introduces the concept of neuro-palliativerehabilitation. Based on this work, the Royal College of Physicians, the National Council for Palliative Care and the British Society of Rehabilitation Medicine have published a national guideline on the interface between neurology, rehabilitation and palliative care. ${ }^{31}$ These guidelines have not yet been fully implemented.

More recently, the Motor Neuron Disease Association has produced the "year of care pathway'32 which describes the specific care and services that may be required with disease progression.

\section{USA}

The current system in the USA is highly decentralised, and there are no national guidelines. Palliative care is not nationally or regionally coordinated but rather consists of a mix of different services which differ by locality. For patients in the community who do not attend multidisciplinary ALS clinics, the availability of specialist palliative care is often limited. Hospice services are organised locally, receive Medicare funding and are geared towards end of life care. Palliative care takes place in the patient's home-approximately $20 \%$ of patients receive hospice services in an inpatient hospice facility. ${ }^{33}$ Home health agencies provide short term care in the patient's home for those patients meeting specific eligibility requirements. Longer term care in the home usually is overseen at the state level but administered locally. Hours of care available and eligibility rules differ from state to state and by the administrating agency.

\section{Germany}

The ALS guidelines of the German Neurological Society ${ }^{34}$ specifically suggest early referral to palliative care. However, in practice, ALS patients are usually managed by their practising 
neurologists and family practitioners. Access to palliative care and hospice services occurs late in the course of the disease, if at all. A network of neuromuscular centres in all major cities offers tertiary care but not all patients are referred to these centres. Although a nationwide network of specialist palliative home care teams has recently been introduced, there is limited collaboration between ALS centres and palliative care teams. The role of the general practitioner in the terminal stages of illness remains underdeveloped. ${ }^{35}$

\section{Australia}

A 'map for integrated care ${ }^{36}$ has been generated, showing the role of key service providers in meeting specific patient needs at different stages. The pathway of the Motor Neuron Disease Association of England, Wales and Northern Ireland was identified as the framework that best describes the triggers for referral, and the role and costs of service providers at specific patient milestones.

\section{Ireland}

There is no established national care pathway nor are there clearly defined triggers for activation of palliative care services for ALS patients. A single multidisciplinary clinic, incorporating palliative care, provides services to up to $80 \%$ of the ALS population. ${ }^{4}$ ALS services are coordinated primarily by hospital based neurologists, with direct referral to community based palliative care services. Management in the later stages of the disease is undertaken collaboratively between the specialist hospital based services and community care, with limited involvement of the general practitioner.

\section{Measurement tools}

Existing and proposed frameworks require rigorous auditing and validation prior to implementation. Because palliative care shifts the emphasis from disease modification to symptom management, outcome measurements are primarily centred around quality of life $(\mathrm{QoL})$ rather than disease progression.

QoL is a concept of emotional, spiritual and physical well being within the individual's specific social, cultural and medical context. Poor quality of life in ALS correlates with hopelessness and lack of social support. ${ }^{37}$ Several studies ${ }^{38-40}$ in ALS have demonstrated that QoL is independent of physical disability and is frequently a function of complex psychosocial and existential/ spiritual factors. This evidence provides the rationale for a $\mathrm{QoL}$ orientated palliative intervention in ALS and measurement of QoL as a marker of successful multidomain palliative care intervention.

Palliative measurement tools can be divided into assessment scales and QoL measurement instruments.

\section{Assessment tools: comprehensive palliative diagnosis}

Formal palliative assessment is particularly important in neurodegenerative disorders where underreporting of pain and discomfort is not uncommon. A structured diagnostic palliative approach identifies areas of distress and discomfort across multiple domains: physical, emotional and spiritual.

The Memorial Symptom Assessment Scale, the Rotterdam Symptom Checklist, the Edmonton Symptom Assessment Scale and the Disability Distress Assessment Tool (DisDAT) ${ }^{41}$ represent some of the more widely used comprehensive assessment tools with potential in non-neoplastic palliative medicine.

DisDAT may be of particular use in neurodegenerative conditions such as ALS, as it is adapted for people with severe communication difficulties. DisDAT documents the patient's own 'vocabulary' of distress signs and behaviours in a so-called 'distress passport'.

\section{Quality of life measures}

QoL measurement in ALS is challenging due to communication barriers. The use of generic instruments (Sickness Impact Profile/ SF-36 Health Survey) is limited in the assessment of QoL in ALS. Memory and cognitive deficits may make testing even more difficult. A number of observational rating scales have been developed to overcome these challenges, many of them using composite scores from carers and patients.

The Schedule for the Evaluation of Individualised Quality of LifeDirect Weighting (SEIQoL-DW) ${ }^{39}$ is a useful tool in ALS, taking individual value priorities into account. In the SEIQoL-DW, patients rate their satisfaction in areas of life which are most important to their QoL. The most relevant domains of QoL in ALS are family, friends/social life, health and profession based on the SEIQoL-DW studies. ${ }^{40}$ Longitudinal QoL studies in ALS demonstrated that while health related $\mathrm{OOL}$ and functional status showed relentless decline, the SEIOOL-DW total score remained stable. Longitudinal studies also concluded that patients' QoL priorities change over time.

The Schedule for Meaning in Life Evaluation (SMiLE) ${ }^{42}$ is used successfully in ALS where satisfaction is weighted against the relevance of individually named domains. The most often highlighted areas of Meaning of Life (MiL) ${ }^{43}$ in ALS were family (82\%), partnership (61\%), leisure activities (53\%) and friends (39\%).

The McGill QoL Questionnaire (MQoL) ${ }^{44}$ addresses five domains: physical well being, physical symptoms, existential well being, psychological symptoms and support through a 20 item questionnaire.

The ALS Specific QoL (ALSSQOL) ${ }^{45}$ rating instrument is based on the MQoL Questionnaire and takes religiousness, spirituality and psychological well being into account.

Evaluating QoL in the presence of cognitive impairment is poorly studied in ALS. In a cross sectional QoL study ${ }^{46}$ of 31 ALS patients, QoL scores correlated positively with social and emotional support and correlated negatively with the presence of everyday cognitive difficulties.

Several QoL scales have been developed for patients with dementia and these could be modified for use in patients with ALS and cognitive impairment. The Psychological Well-Being in Cognitively Impaired Persons, ${ }^{47}$ the Dementia Care Mapping ${ }^{48}$ and the the Cornell-Brown Scale for Quality of Life in Dementia ${ }^{49}$ are either observer rated assessment instruments or incorporate the caregiver's perspective.

The use of these instruments in ALS is currently limited and many of these tests were developed and validated for Alzheimer's dementia rather than the frontotemporal cognitive deficits typical of ALS. However, with the emerging evidence of relatively high incidence of cognitive impairment in ALS, the development of ALS specific QoL instruments for patients with cognitive impairment is desirable.

\section{Common themes of international initiatives}

The appropriate timing of palliative intervention is essential and confers important quality of life benefits. Despite cultural and financial differences worldwide, a number of common principles can be identified to ensure high quality palliative care in ALS (box 5).

\section{CONCLUSIONS}

ALS management requires clear communication and cooperation between different medical specialties, hospital and community 
Box 5 Common themes of palliative initiatives in amyotrophic lateral sclerosis care

- Requirement for a national framework and patient pathway

- Nomination of a single person to coordinate amyotrophic lateral sclerosis care in a given phase of the disease

- Multidisciplinary amyotrophic lateral sclerosis care (incorporating hospital and community based palliative care services)

- Formal cognitive testing prior to advance directives

- Early and local palliative care involvement

- Structured caregiver support (grief support, counselling, support groups, crisis management system)

- Qualitative and quantitative measurement scheme to assess benefits of palliative care interventions

- Need for development of international consensus guidelines for the palliative management of amyotrophic lateral sclerosis

based services. Patients and caregivers should be linked in early to specialist palliative care services through multidisciplinary ALS clinics. A dynamic, evidence based framework for integrating palliative care into the management of ALS is urgently required, and would be best achieved in the context of an international consensus. Effective symptom management, advance discussion of care preferences and continued caregiver support are important governing principles. Disease specific quality of life measurement tools should be applied to assess and validate the role of palliative intervention in ALS. A successful palliative model in ALS will in turn assist in the development of palliative care models for other neurodegenerative conditions.

\section{Competing interests None}

Provenance and peer review Not commissioned; externally peer reviewed.

\section{REFERENCES}

1. Veronese $\mathbf{S}$, Gallo G, Valle A, et al. Specialist palliative care service for people severely affected by neurodegenerative conditions: does this make a difference to palliative care outcomes? Results of Nepal-an explorative randomized controlled trial. Palliat Med 2010;24(Suppl 4):S25-6.

2. Chio A, Mora G, Balzarino C, et al. Interdisciplinary ALS Centres: effect of survival and use of health services in a population-based survey. Neurology 2004;62(Suppl 5):S23.003.

3. Van den Berg JP, Kalmijn S, Lindeman E, et al. Multidisciplinary ALS care improves quality of life in patients with ALS. Neurology 2005;65:1264-7.

4. Traynor BJ, Alexander M, Corr B, et al. Effect of a multidisciplinary amyotrophic lateral sclerosis (ALS) clinic on ALS survival: a population based study, 1996-2000. J Neurol Neurosurg Psychiatry 2003;74:1258-1.

5. Ganzini L, Johnston WS, Silveira MJ. The final month of life in patients with ALS. Neurology 2002;59:428-31.

6. Andersen PM, Borasio GD, Dengler R, et al. EFNS task force on management of amyotrophic lateral sclerosis: guidelines for diagnosing and clinical care of patients and relatives; An evidence-based review with good practice points. Eur J Neurol 2005;12:921-38.

7. Borasio GD, Shaw PJ, Hardiman 0, et al. Standards of palliative care for patients with amyotrophic lateral sclerosis: results of a European survey. Amyotroph Lateral Scler 2001;2:159-64.

8. Oliver D, Webb S. The involvement of specialist palliative care in the care of people with motor neuron disease. Palliat Med 2000;14:427-8.

9. Elman LB, Houghton DJ, Wu GF, et al. Palliative care in amyotrophic lateral sclerosis, Parkinson's disease, and multiple sclerosis. J Palliat Med 2007;10:433-57.

10. McCluskey L, Houseman G. Medicare hospice referral criteria for patients with amyotrophic lateral sclerosis: a need for improvement. J Palliat Med 2004; 7:47-53

11. Mitsumoto $\mathbf{H}$, Bromberg $\mathbf{M}$, Johnston $\mathbf{W}$, et al. Promoting excellence in end-of-life care in ALS. Amyotroph Lateral Scler 2005;6:145-54.

12. Hardiman 0, Traynor BJ, Corr B, et al. Models of care in motor neurone disease: setting standards. Amyotroph Lateral Scler Other Motor Neuron Disord 2002;3:182-218.
13. Corr B, Frost E, Markey $F$, et al. Access to palliative care services by Irish ALS patients. ALS J 2003;4:219-20.

14. O'Leary N, Tiernan E. Survey of specialist palliative care services for noncancer patients in Ireland and perceived barriers. Palliat Med 2008;22:77-83.

15. Oliver D, Campbell C, Sloan R, et al. End-of-life care and decision making in ALS/ MND: A crosscultural study. Amyotroph Lateral Scler 2007;8(Suppl 1):13-15.

16. Anon. Completing the Continuum of ALS Care: A Consensus Document. Missoula (MT), USA: The Robert Wood Johnson Foundation, Missoula (MT), USA, 2004.

17. Hebert RS, Lacomis D, Easter $C$, et al. Grief support for informal caregivers of patients with ALS: A national survey, Neurology 2005;64:137-8.

18. Kapo J, Harrold J, Carrol J, et al. Are we referring patients to hospice too late? J Palliat Med 2005;8:521-7.

19. Strong $\mathbf{M}$. The syndromes of frontotemporal dysfunction in amyotrophic lateral sclerosis. Amyotroph Lateral Scler 2008;9:323-38.

20. Phukan J, Pender NP, Hardiman 0. Cognitive impairment in amyotrophic lateral sclerosis. Lancet Neurol 2007;6:994-1003.

21. Strong $\mathbf{M}$, Grace GM, Freedman $\mathbf{M}$, et al. Consensus criteria for the diagnosis of frontotemporal cognitive and behavioural syndromes in amyotrophic lateral sclerosis. Amyotroph Lateral Scler 2009;10:131-46.

22. Goldstein L. Control of symptoms: cognitive dysfunction. In: Oliver D, Borasio D, Walsh D. Palliative Care in Amyotrophic Lateral Sclerosis from Diagnosis to Bereavement. 2nd edn. Oxford: Oxford University Press, 2006:111-27.

23. Flaherty-Craig C, Eslinger $P$, Stephens $B$, et al. A rapid screening battery to identify frontal dysfunction in patients with ALS. Neurology 2006;67:2070-2.

24. OIney RK, Murphy J, Forshew D, et al. The effects of executive and behavioral dysfunction on the course of ALS. Neurology 2005;65:1774-7.

25. Rabkin J, Albert SM, Tider T, et al. Predictors and course of elective long-term mechanical ventilation: A prospective study of ALS patients. Amyotroph Lateral Scler 2006; 7:86-95.

26. Chiò A, Vignola A, Mastro E, et al. Neurobehavioral symptoms in ALS are negatively related to caregivers' burden and quality of life. Eur J Neurol 2010;17:1298-303

27. Oliver DJ, Turner MR. Some difficult decisions in ALS/MND. Amytroph Lateral Scler 2010;11:339-43.

28. Mitsumoto H, Rabkin JG. Palliative care for patients with amyotrophic lateral sclerosis: "prepare for the worst and hope for the best". JAMA 2007;298:207-16.

29. Van den Berg JP, de Groot IJM, Joha BC, et al. Development and implementation of the Dutch protocol for rehabilitative management in amyotrophic lateral sclerosis. Amyotroph Lateral Scler Other Motor Neuron Disord 2004;5:226-9.

30. Brown J, Sutton L. Pathway Leading to the Neurological Diagnosis, Neurological Care Pathway. NCPC - National Council for Palliative Care, Focus on Neurology, 2007. This is a publication of the National Council for Palliative Care in the UK and the NHS in the UK. It is available online at: http://www.endoflifecareforadults.nhs.uk/ assets/downloads/neurology_report__ final__20101108_1.pdf laccessed 7 Jan 2011).

31. NCPC. Neurological Conditions from Diagnosis to Death-Exploring the Interface Between Palliative Care, Neurology and Rehabilitation in the Management of People With Long Term Neurological Conditions. NCPC - National Council for Palliative Care, National Survey. This is a publication of the National Council for Palliative Care in the UK (www.ncpc.org.uk), ISBN: 189891550 4, published June 2006.

32. End of life care in neurological conditions: a framework for implementation. National End of Life Programme 2010. MND, Association Year of Care Pathway. 2008. This is a publication of the National Council for Palliative Care in the UK and the NHS in the UK. It is available online at: http://www.endoflifecareforadults.nhs.uk/ assets/downloads/neurology_report__ final__20101108_1.pdf laccessed 7 Jan 2011).

33. National Hospice and Palliative Care Organisation (NHPCO). Facts and Figures: Hospice Care in America. National Hospice and Palliative Care Organisation (NHPCO), 2009. Available online at: http://www.nhpco.org/files/public/ Statistics_Research/NHPCO_facts_and_figures.pdf (accessed 7 Jan 2011).

34. Anon. Guideline on ALS by the German Neurological Society. 2008; (in German): http://www.dgn.org/images/stories/dgn/leitlinien/LL2008/I08kap_019.pdf (accessed 7 Jan 2011).

35. Kühnlein P, Kübler A, Raubold S, et al. Palliative care and circumstances of dying in German ALS patients using non-invasive ventilation. Amyotroph Lateral Scler 2008:9:91-8.

36. Anon. Motor Neurone Disease and palliative care, Interim Report on the MND Pathway Project. Melbourne, Victoria, Australia: Metropolitan Health and Aged Care Services Division, Victorian Government Department of Human Services, 2008.

37. Ganzini L, Johnston JS, Hoffman WF. Correlates of suffering in amyotrophic lateral sclerosis. Neurology 1999;52:1434-40.

38. Simmons Z, Bremer BA, Robbins RA, et al. Quality of life in ALS depends on factors other than strength and physical function. Neurology 2000;55:388-92.

39. O'Doherty L, Hickey A, Hardiman O. Measuring life quality, physical function and psychological well-being in neurological illness. Amyotroph Lateral Scler 2010;11:461-8.

40. Neudert C, Wasner M, Borasio GD. Individual quality of life is not correlated with health-related quality of life or physical function in patients with amyotrophic lateral sclerosis. J Palliat Med 2004;7:551-7.

41. Regnard C, Reynolds J, Watson B, et al. Understanding distress in people with severe communication difficulties: developing and assessing the Disability Distress Assessment Tool (DisDAT). J Intellect Disabil Res 2007:51:277-92. 
42. Fegg MJ, Kramer M, L'hoste S, et al. The Schedule for Meaning in Life Evaluation (SMiLE): validation of a new instrument for meaning-in-life research. J Pain Symptom Manage 2008;35:356-64.

43. Fegg MJ, Kögler M, Brandstätter $\mathrm{M}$, et al. Meaning in life in patients with amyotrophic lateral sclerosis. Amyotroph Lateral Scler 2010;11:469-74.

44. Cohen R, Mount BM, Strobel MG, et al. The McGill Quality of Life Questionnaire: a measure of quality of life appropriate for people with advanced disease. A preliminary study of validity and acceptability. Palliat Med 1995;9:207-19.

45. Simmons Z, Felgoise SH, Bremer BA, et al. The ALSSOOL-Balancing physical and nonphysical factors in assessing quality of life in ALS. Neurology 2006;67:1659-64.
46. Goldstein LH, Atkins L, Leigh PN. Correlates of quality of life in people with motor neuron disease (MND). Amyotroph Lateral Scler Other Motor Neuron Disord 2002; $3: 123-9$.

47. Burgener S, Twigg P, Popovich A. Measuring psychological well-being in cognitively impaired persons. Dementia 2005; 4:463-85.

48. Brooker DJ, Surr C. Dementia Care Mapping (DCM): initial validation of DCM 8 in UK field trials. Int J Geriatr Psychiatry 2006;21:1018-25.

49. Ready RE, Ott BR, Grace J, Fernandez I. The Cornell-Brown Scale for Quality of Life in dementia. Alzheimer Dis Assoc Disord 2002;16:109-15.

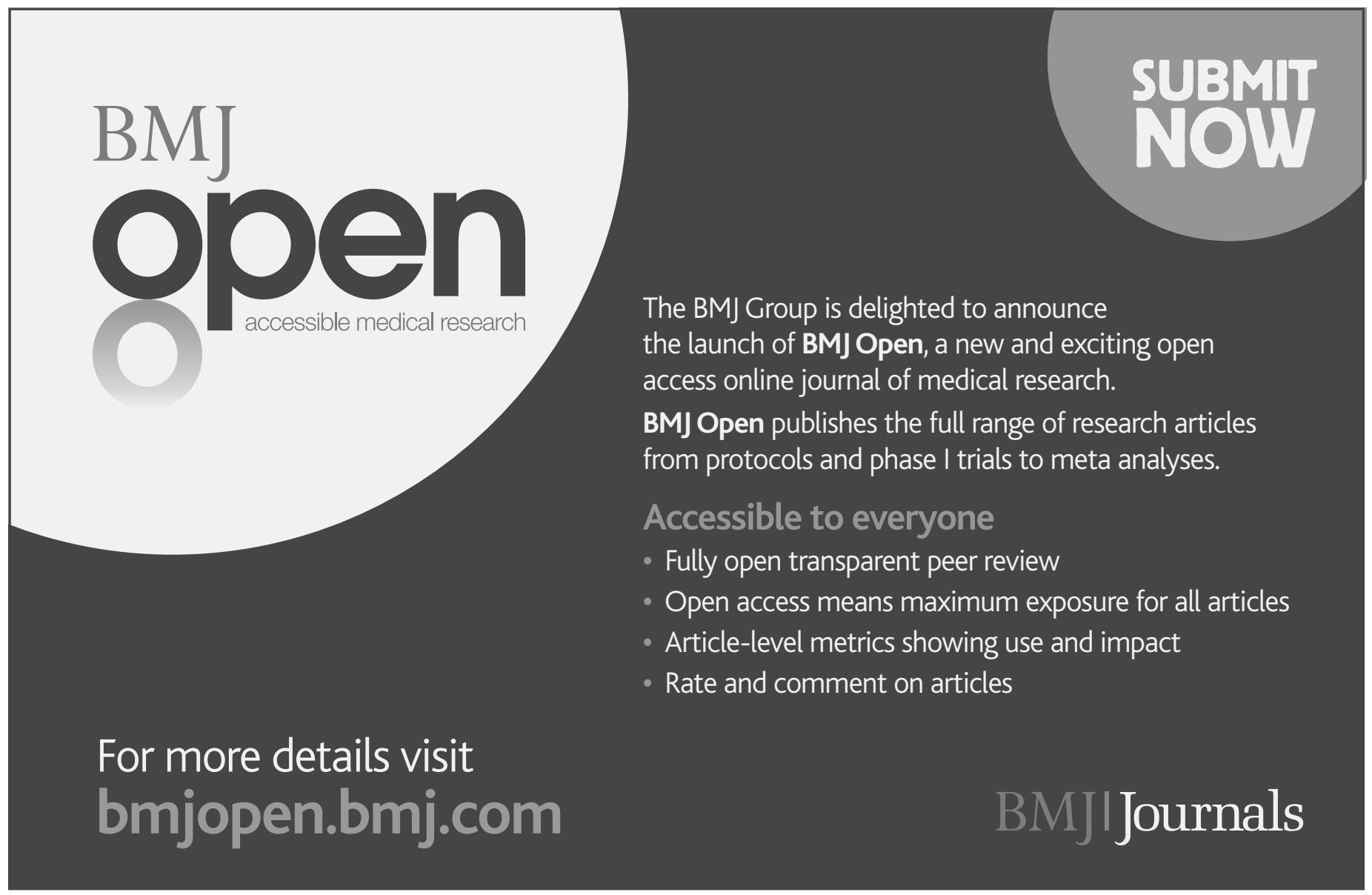



sclerosis: a review of current international guidelines and initiatives

Peter Bede, David Oliver, James Stodart, Leonard van den Berg, Zachary Simmons, Doiminic Ó Brannagáin, Gian Domenico Borasio and Orla Hardiman

J Neurol Neurosurg Psychiatry 2011 82: 413-418 originally published online February 5, 2011

doi: 10.1136/jnnp.2010.232637

Updated information and services can be found at:

http://jnnp.bmj.com/content/82/4/413

\section{These include:}

References This article cites 41 articles, 13 of which you can access for free at: http://jnnp.bmj.com/content/82/4/413\#BIBL
Email alerting service
Receive free email alerts when new articles cite this article. Sign up in the box at the top right corner of the online article.

Topic Articles on similar topics can be found in the following collections

Collections

Hospice (26)

Motor neurone disease (252)

Neuromuscular disease (1175)

Spinal cord (467)

Drugs: CNS (not psychiatric) (1734)

\section{Notes}

To request permissions go to:

http://group.bmj.com/group/rights-licensing/permissions

To order reprints go to:

http://journals.bmj.com/cgi/reprintform

To subscribe to BMJ go to:

http://group.bmj.com/subscribe/ 